\title{
Two trends of sample dispersion variation with carrier flow rate in a single flow-injection manifold
}

\author{
Yonghung Li, Huichang Ma * \\ Research Center for Eco-Environmental Sciences, Academia Sinica, P.O. Box 2871, Beijing 100085, China
}

Received 3 January 1995; revised 7 July 1995; accepted 17 July 1995

\begin{abstract}
Two trends of sample dispersion variation with carrier flow rate in a single flow-injection manifold are completely revealed for the first time and an inflection point in the dispersion coefficient $(D)$ vs. flow rate $(q)$ curve is discovered. With the increase of the flow rate, the value of $D$ increases before the inflection point but decreases after the inflection point. The value of the carrier flow rate at the inflection point $\left(q_{m}\right)$ is independent of the sample injection volume, the tube length, the tube coil radius and the tube inner diameter. It is only affected by the substance diffusion coefficient $\left(D_{\mathrm{m}}\right)$ of the analysis. The value of $q_{\mathrm{m}}$ decreases as $D_{\mathrm{m}}$ increases. Therefore, the value of $D_{\mathrm{m}}$ for a sample can be estimated according to the $D_{\mathrm{m}}$ vs. $q_{\mathrm{m}}$ curve.
\end{abstract}

Keywords: Sample dispersion variation; Carrier flow rate; Flow-injection manifold

\section{Introduction}

In the flow injection analysis (FIA), the dispersion coefficient $(D)$ is defined as $D=C^{0} / C$, i.e. as the ratio of the concentrations before and after the dispersion process has taken place in the individual elements of fluid. If a readout is taken of $C=C^{\max }$, i.e. the element corresponding to the maximum on the output curves, then $D=C^{0} / C^{\max }$. Since the dispersion coefficient represents the degree of dilution of the sample, the value of $D$ directly affects the sensitivity of any flow injection assay. Therefore, the study of the sample dispersion coefficient is very important in basic FIA research.

It is well known in FIA that the factors predominantly affecting the sample dispersion are the sample injection volume $\left(V_{\mathrm{inj}}\right)$, the tube length $(L)$ and inner diameter $(d)$, the tube coil

- Corresponding author. radius $\left(R_{\mathrm{c}}\right)$, the carrier flow rate $(q)$, the diffusion coefficient of the analyte $\left(D_{\mathrm{m}}\right)$ and temperature. The originators of FIA, Ruzicka and Hansen [1], determined that the value of $D$ decreases with decreasing flow rate in a single flow injection manifold. However, $\mathrm{Li}$ and Narusawa [2] demonstrated in their experiments that the value of $D$ gradually increases with a decrease in flow rate. Gomez-Nieto et al. [3] gave an empirical formula, $D=$ $2.342 L^{0.167} q^{-0.206} d^{0.495}$, which indicates that $D$ is inversely proportional to the flow rate. Thus, there are two completely different opinions. Up until now, no satisfactory explanation has been given for this.

Since flow rate is an important factor which affects the dispersion coefficient, this paper will investigate and experimentally test these two opinions and try to offer a reasonable explanation, which it is hoped will be instructive to experimentalists who wish to design flow injection systems. 


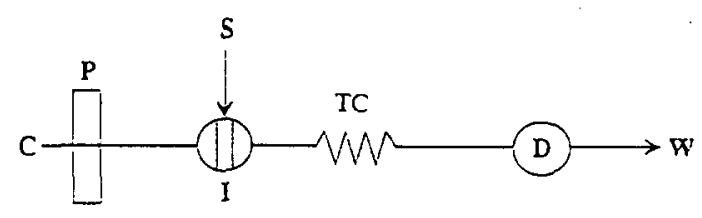

Fig. 1. Manifold for the single flow injection system: $\mathbf{P}$, peristaltic pump; I, injection value; TC, tube coil (made of PTFE); D, detector (with a flow cell of $18 \mu \mathrm{l}$ and $10 \mathrm{~mm}$ pathlength); $S$, sample; $C$, carrier; $W$, waste.

\section{Experimental}

\subsection{Reagents}

Aqueous solutions of $1.59 \times 10^{-2} \mathrm{M}$ $\mathrm{Cu}\left(\mathrm{NH}_{3}\right)_{4}^{2+}$ in $1.0 \mathrm{M}$ ammonia and $1.0 \mathrm{M}$ ammonia were mainly used as sample and carrier respectively. The sample solution was prepared by dissolving $0.400 \mathrm{~g} \mathrm{CuSO}_{4} \cdot 5 \mathrm{H}_{2} \mathrm{O}$ in $100 \mathrm{ml}$ of $1.0 \mathrm{M}$ ammonia. Other colored inorganic chemicals such as $\mathrm{K}_{3} \mathrm{Fe}(\mathrm{CN})_{6}$ and $\mathrm{K}_{2} \mathrm{Cr}_{2} \mathrm{O}_{7}$ were also used as samples.

All reagents used were of analytical reagent grade and were obtained from Peking Chemical Works, Peking, China. All solutions were prepared with distilled water.

\subsection{Apparatus}

An FIA-91 flow injection analyzer (made in Shanghai No. 3 Analytical Instrument Factory) was used. This is an integrated instrument which consists of two four-channel peristaltic pumps, a 16-port valve, a grating spectrophotometric detector, a Laser PP40 X-Y printer plotter and a microcomputer system. Program control and data processing are conducted automatically by the microcomputer system.

\subsection{Procedure}

The manifold of the single channel FIA system used is shown in Fig. 1. As can be seen from Fig. 1, after the sample is injected it is forwarded by the carrier to pass through a certain length of coiled tube and eventually to the spectrophotometric detector. For different values of $V_{\mathrm{inj}}, L$, $R_{\mathrm{c}}, d$ and $D_{\mathrm{m}}$, the effect of flow rate on sample dispersion is observed.

\section{Results and discussion}

\subsection{Effect of flow rate on dispersion coefficient with different injection volumes}

In these experiments, an aqueous solution of $1.59 \times 10^{-2} \mathrm{M} \mathrm{Cu}\left(\mathrm{NH}_{3}\right)_{4}^{2+}$ in $1.0 \mathrm{M}$ ammonia as spiked sample and an aqueous solution of $1.0 \mathrm{M}$ ammonia as carrier were used. Selected results are listed in Table 1 and a group of $D$ vs. $q$ curves are shown in Fig. 2. The correlations between residence time $\left(T_{\mathrm{r}}\right)$ and flow rate are listed in Table 2. From Table 1 it can be seen that the residence time increases with increasing injection volume $\left(V_{\text {inj }}\right)$ while the dispersion coefficient decreases. For identical values of $V_{\text {inj }}$, $T_{\mathrm{r}}$ decreases with increasing flow rate.

From Fig. 2 it is obvious that there is an inflection point (where $\mathrm{d} D / \mathrm{d} q=0$ ) in the $D$ vs. $q$ curve. With increasing flow rate the value of $D$ increases before this point but decreases after this point. The value of $q$ at the inflection point $\left(q_{\mathrm{m}}\right)$ is independent of the injection volume.

In a single FIA system, $T_{\mathrm{r}}$ decreases as $q$ increases. Ruzicka and Hansen [4] have given a theoretical formula, $D=2 q T_{\mathrm{r}}^{1 / 2} t_{\mathrm{i}}^{1 / 2} / W_{0} \pi(d / 2)^{2}$, where $t_{\mathrm{i}}$ is the mean residence time and $W_{0}$ is

Table 1

Experimental data obtained for the determination of $\mathrm{Cu}\left(\mathrm{NH}_{3}\right)_{4}^{2+}$ dispersion

\begin{tabular}{|c|c|c|c|c|c|c|c|c|c|c|}
\hline \multirow[t]{3}{*}{$q(\mathrm{ml} \mathrm{min}-1)$} & \multicolumn{10}{|c|}{$V_{\text {inj }}(\mu l)$} \\
\hline & \multicolumn{2}{|l|}{40} & \multicolumn{2}{|l|}{80} & \multicolumn{2}{|l|}{120} & \multicolumn{2}{|l|}{160} & \multicolumn{2}{|l|}{180} \\
\hline & $T_{\mathrm{r}}(\mathrm{s})$ & $D$ & $T_{\mathrm{r}}(\mathrm{s})$ & $D$ & $T_{\mathrm{r}}(\mathrm{s})$ & $D$ & $T_{\mathrm{r}}$ (s) & $D$ & $T_{\mathrm{r}}(\mathrm{s})$ & $D$ \\
\hline 1.22 & 41.44 & 4.02 & 42.38 & 2.36 & 44.06 & 1.79 & 45.50 & 1.53 & 47.02 & 1.38 \\
\hline 1.53 & 33.62 & 4.14 & 34.37 & 2.43 & 35.71 & 1.83 & 36.89 & 1.55 & 37.91 & 1.39 \\
\hline 2.48 & 21.72 & 4.67 & 22.22 & 2.64 & 23.00 & 1.93 & 23.60 & 1.62 & 24.40 & 1.43 \\
\hline 3.23 & 17.60 & 5.23 & 18.06 & 2.94 & 18.50 & 2.13 & 19.28 & 1.76 & 20.28 & 1.56 \\
\hline 4.29 & 12.66 & 4.89 & 13.20 & 2.82 & 13.62 & 2.00 & 14.02 & 1.70 & 14.10 & 1.48 \\
\hline 5.45 & 10.16 & 4.20 & 10.43 & 2.53 & 10.86 & 1.89 & 11.16 & 1.57 & 11.48 & 1.40 \\
\hline 7.14 & 7.56 & 3.71 & 7.86 & 2.26 & 8.19 & 1.78 & 8.34 & 1.57 & 8.38 & 1.40 \\
\hline
\end{tabular}




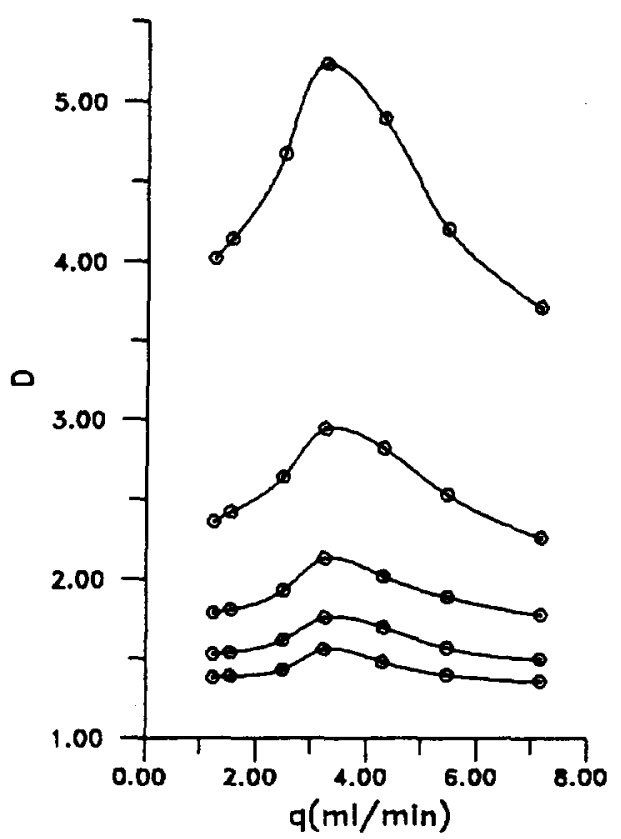

Fig. 2. The $D$ vs. $q$ curves with various injection volumes (tube: $L=233 \mathrm{~cm} ; d=0.5 \mathrm{~mm} ; R_{\mathrm{c}}=1.0 \mathrm{~cm}$ ).

Table 2

The equations of $\lg T_{\mathrm{r}}=a \lg q+b \quad(r=$ correlation coefficient)

\begin{tabular}{|c|c|c|c|c|}
\hline \multirow[t]{2}{*}{$V_{\text {inj }}(\mu l)$} & \multicolumn{2}{|c|}{ Before inflection point } & \multicolumn{2}{|c|}{ After inflection point } \\
\hline & $a$ & $r$ & $a$ & $r$ \\
\hline 40 & -0.886 & -0.9996 & -1.052 & -0.9978 \\
\hline 80 & -0.884 & -0.9995 & -1.042 & -0.9940 \\
\hline 120 & -0.896 & -0.9998 & -1.021 & -0.9993 \\
\hline 160 & -0.891 & -0.9993 & -1.047 & -0.9989 \\
\hline 200 & -0.879 & -0.9985 & -1.091 & -0.9941 \\
\hline
\end{tabular}

independent of $q$ and $t$. It is known that $t_{\mathrm{i}}=k L / q \quad(k=\mathrm{constant})$. Thus, $D+f\left(q T_{r}\right)^{1 / 2}$ $(f=$ constant $)$. If $T_{\mathrm{r}}=f q^{-1} \quad(f=$ constant $)$, the two effects, with the inverse changes of $T_{\mathrm{r}}$ and $q$ on the dispersion coefficient, can offset each other. However, Table 2 shows that $T_{\mathrm{r}}=f q^{-0.886}\left(f=\right.$ constant, $\left.a_{\text {mean }}=-0.886\right)$ before the inflection point. This means that $T_{\mathrm{r}}$ decreases more slowly with increasing flow rate than is to be expected. The relative longer $T_{r}$ time makes $D$ increase with the increase in flow rate. However, after the inflection point, $T_{\mathrm{r}}=f q^{-1.051} \quad\left(f=\right.$ constant,$\left.\quad a_{\text {mean }}=-1.051\right)$ which means that $T_{\mathrm{r}}$ decreases more quickly with increasing flow rate than expected. The relative shorter $T_{\mathrm{r}}$ time makes $D$ decrease with the increase in flow rate. Treating the data in the paper of Li and Narusawa [2] statistically, we obtained $T_{\mathrm{r}}=f q^{-1.018} \quad(f=$ constant, $r=-0.9991$ ). From Gomez-Nieto et al. [3] we can show that the relationship between $T_{r}$ and $q$ is $T_{\mathrm{r}}=f q^{-1.022} \quad(f=$ constant, $r=-0.9993)$ when $d=0.35 \mathrm{~mm}$ and $L=104 \mathrm{~cm}$ or when $d=0.5 \mathrm{~mm}$ and $L=154 \mathrm{~cm}$. The indices of $q$ are both smaller than -1.0 while these two papers have the same conclusion, i.e. that $D$ gradually decreases as $q$ increases.

Thus, it is clear that the view of Ruzicka and Hansen [4] coincides with the part before the inflection point while of view of $\mathrm{Li}$ and Narusawa [2] coincides with the part after the inflection point in this experiment. Therefore, this experiment has clearly revealed the two trends of sample dispersion variation with flow rate.

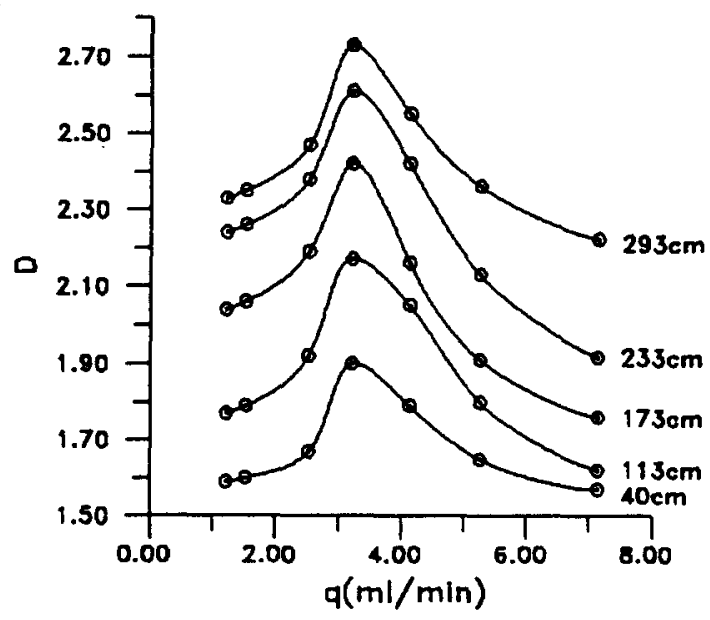

Fig. 3. The $D$ vs. $q$ curves with various tube lengths $\left(V_{\text {inj }}=80 \mu \mathrm{l}\right.$; tube: $\left.d=0.5 \mathrm{~mm} ; R_{\mathrm{c}}=1.0 \mathrm{~cm}\right)$.

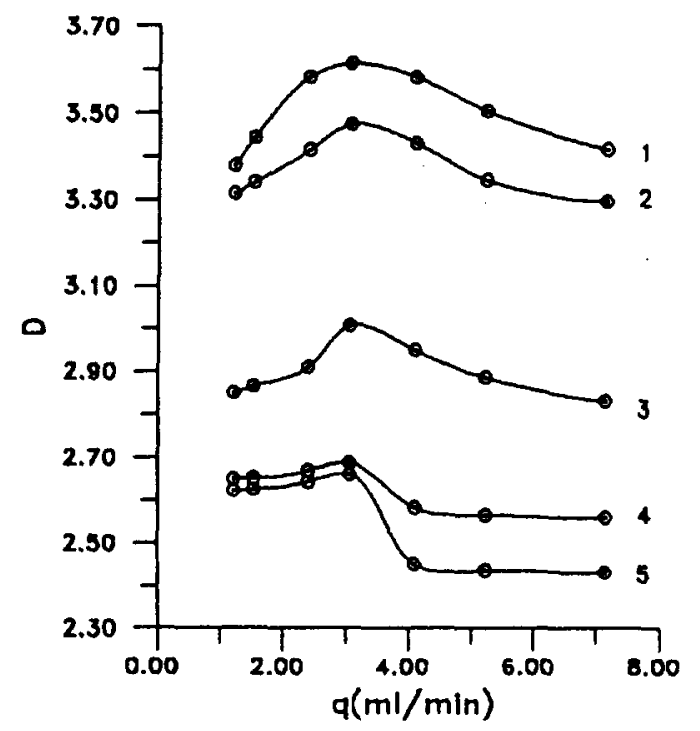

Fig. 4. The $D$ vs. $q$ curves with various tube coil radii ( $V_{\text {inj }}=80 \mu$ l, tube: $\left.L=233 \mathrm{~cm} ; d=0.5 \mathrm{~mm}\right) . R_{\mathrm{c}}(\mathrm{cm})$ : (1), 0.5 ; (2), 1.0; (3), 1.5; (4), 2.0; (5), 2.5. 


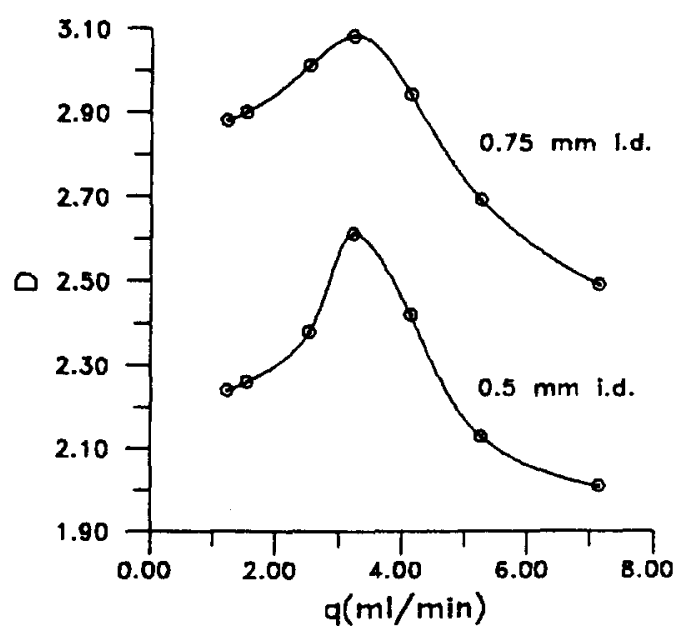

Fig. 5. The $D$ vs. $q$ curves with various tube inner diameters $\left(V_{\text {inj }}=80 \mu\right.$ l, tube: $L=233 \mathrm{~cm} ; R_{\mathrm{c}}=1.0 \mathrm{~cm}$ ).

\subsection{Effect of tube length $(L)$ on the $D$ vs. $q$ curve}

The effect of tube length on the correlation between $D$ and $q$ was examined in the range $40-293 \mathrm{~cm}$. Using the same sample and carrier as before, the injection volume was fixed at $V_{\text {inj }}=80 \mu \mathrm{l}$. The results obtained are shown in Fig. 3 which indicates that the value of $D$ increases as $L$ increases at the same flow rate. This is because of longer dispersion times caused by longer tube lengths. According to Fig. 3, it is evident that there is an inflection point in the $D$ vs. $q$ curve at different tube lengths and the value of $q_{\mathrm{m}}$ is constant even as the tube length changes.

\subsection{Effect of tube coil radius on the $D$ vs. $q$ curve}

To test the effect of tube coil radius $\left(R_{\mathrm{c}}\right)$, tube coils of various radii were used. Using the same sample and carrier as before, the FIA system was fixed at $V_{\text {inj }}=80 \mu l$, tube length $L=233 \mathrm{~cm}$. From the results shown in Fig. 4 , it is obvious that the value of $D$ increases as the tube coil radius decreases. The results also indicated that there is a inflection point and that the value of $q_{m}$ remains the same as the tube coil radius varies.

\subsection{Effect of tube inner diameter on the $D$ vs. $q$ curve}

The effect of tube inner diameter was inves- tigated by using different inner diameter tubes. The same sample and carrier as before were used and the injection volume was fixed at $V_{\text {inj }}=80 \mu \mathrm{l}$ while the tube length was $L=233 \mathrm{~cm}$. From the results shown in Fig. 5 it can be seen that $D$ increases as $d$ increases which is identical to former conclusions. The value of $q_{\mathrm{m}}$ remains the same as $d$ varies.

\subsection{Effect of the analyte coefficient on the $D$ vs. q curve}

To test the effect of $D_{\mathrm{m}}$, various samples and their corresponding carriers as listed in Table 3 were used. The flow injection manifold was fixed at $V_{\text {inj }}=80 \mu \mathrm{l}, L=233 \mathrm{~cm}$ and $d=0.5 \mathrm{~mm}$. The experimental temperature was about $25^{\circ} \mathrm{C}$. The results are shown in Fig. 6.

From Fig. 6 it is obvious that the value of $q_{\mathrm{m}}$ decreases while $D_{\mathrm{m}}$ increases and that $D$ increases with increasing $D_{\mathrm{m}}$ while the flow rate is constant.

It is well known that in a single channel FIA system, the sample dispersion during the flow towards the detector is composed of two parts: one is the convection dispersion which is caused by the flow of the carrier and is directly proportional to the value of the flow rate; the other is the diffusion dispersion which is caused by the diffusion of the analyte and is directly proportional to the diffusion coefficient of the analyte. When $V_{\mathrm{inj}}, L, R_{\mathrm{c}}, d$ and $q$ are kept constant, the convection dispersion part remains constant. Therefore, higher dispersion can be obtained because of increased diffusion dispersion of the analyte, i.e. the value of $D$ increases.

It has been shown that before the inflection point, the dispersion increases because of the increase in $q$ and the relatively slower decrease of $T_{r}$. However, the diffusion dispersion part decreases more quickly for the larger $D_{\mathrm{m}}$ value of the analyte as $T_{r}$ decreases. Therefore, the value of $q_{\mathrm{m}}$ is smaller when the $D_{\mathrm{m}}$ value of the analyte is larger (Fig. 7).

\section{Conclusions}

The experiments in this paper revealed the law of the variation of sample dispersion coefficient with carrier flow rate in single coiled 
Table 3

Samples and carriers used to test the effect of analyte diffusion coefficient on the $D$ vs. $q$ curve (temperature $=25^{\circ} \mathrm{C}$ )

\begin{tabular}{lll}
\hline Sample & Carrier & $\begin{array}{l}\text { Diffusion coefficient } \\
\left(\times 10^{-5} \mathrm{~cm}^{2} \mathrm{~s}^{-1}\right)\end{array}$ \\
\hline $4.56 \times 10^{-3} \mathrm{M} \mathrm{K}_{3} \mathrm{Fe}(\mathrm{CN})_{6}$ in $1.0 \mathrm{M} \mathrm{NaOH}^{\mathrm{a}}$ & $1.0 \mathrm{M} \mathrm{NaOH}$ & 0.45 \\
$2.47 \times 10^{-2} \mathrm{M} \mathrm{CuSO}_{4}$ in $0.2 \mathrm{M} \mathrm{H}_{2} \mathrm{SO}_{4}{ }^{\mathrm{b}}$ & $0.2 \mathrm{M} \mathrm{H} \mathrm{SO}_{4}$ & 0.57 \\
$4.56 \times 10^{-3} \mathrm{M} \mathrm{K}_{3} \mathrm{Fe}(\mathrm{CN})_{6}$ in $1.0 \mathrm{M} \mathrm{KCl}^{\mathrm{a}}$ & $1.0 \mathrm{M} \mathrm{KCl}$ & 0.76 \\
$4.56 \times 10^{-3} \mathrm{M} \mathrm{K}_{3} \mathrm{Fe}(\mathrm{CN})_{6}{ }^{\mathrm{c}}$ & $\mathrm{H}_{2} \mathrm{O}$ & 0.96 \\
$6.80 \times 10^{-4} \mathrm{M} \mathrm{K}_{2} \mathrm{Cr}_{2} \mathrm{O}_{7}{ }^{\mathrm{C}}$ & $\mathrm{H}_{2} \mathrm{O}$ & 1.24 \\
\hline
\end{tabular}

"Ref. [5]. ${ }^{\circ}$ Ref. [6]. ${ }^{c}$ Ref. [7].

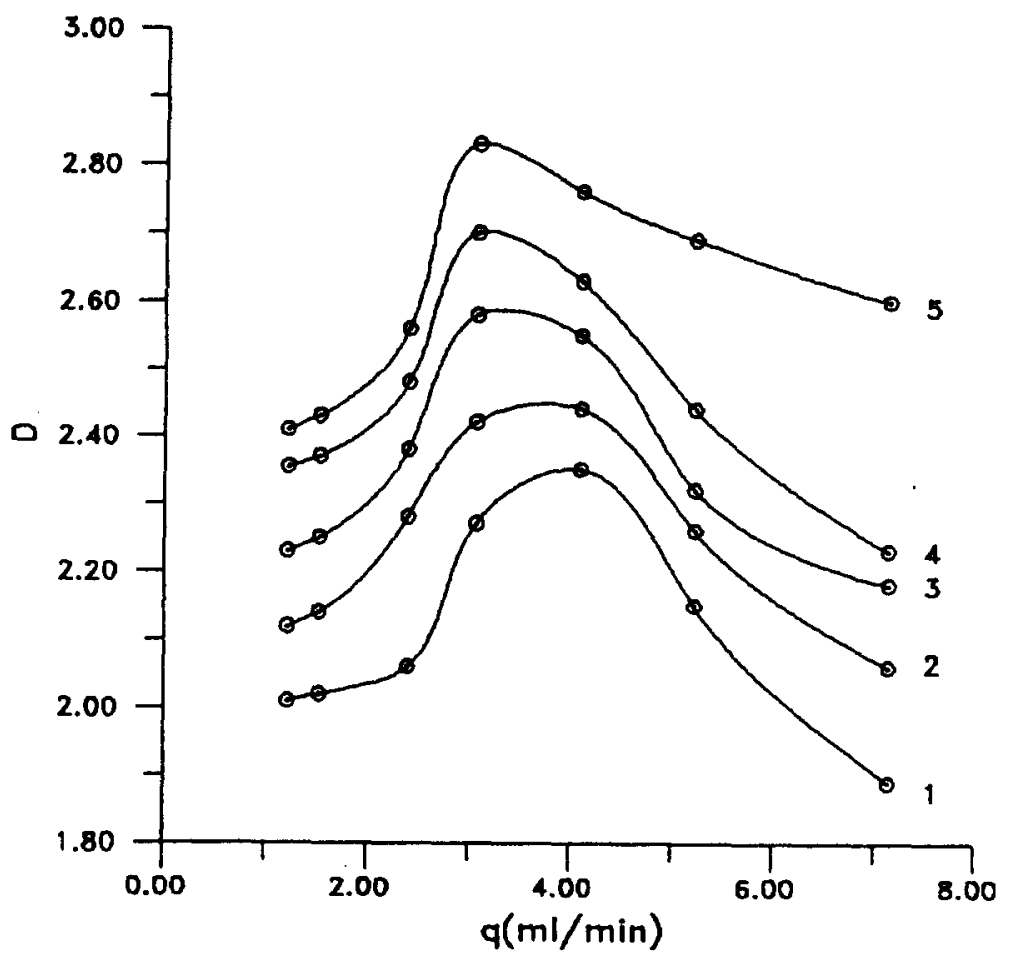

Fig. 6. The $D$ vs. $q$ curves with various analyte diffusion coefficients $\left(V_{\text {inj }}=80 \mu \mathrm{l}\right.$, tube: $L=233 \mathrm{~cm} ; d=0.5 \mathrm{~mm}$; $\left.R_{\mathrm{c}}=1.0 \mathrm{~cm}\right) . D_{\mathrm{m}}\left(\times 10^{-5} \mathrm{~cm}^{2} \mathrm{~s}^{-1}\right.$; (1), 0.45; (2), 0.57; (3), 0.76; (4), 0.96; (5), 1.24.

tube channel FIA systems and yield the following guidelines which should be useful to experimentalists who wish to design FIA systems.

(1) There is an inflection point (where $\mathrm{d} D /$ $\mathrm{d} q=0$ ) in the $D$ vs. $q$ curve.

(2) With the increase in the flow rate, the value of $D$ increases before the inflection point and decreases after this point.

(3) The value of $q$ at the inflection point is independent of the sample injection volume, the tube length, the tube coil radius and the inner diameter of the tube. It is only affected by the diffusion coefficient of the analyte.

(4) The value of $q_{m}$ decreases while $D_{m}$ increases. According to the $q_{\mathrm{m}}$ vs. $D_{\mathrm{m}}$ curve, the $D_{m}$ value of an unknown analyte can be roughly estimated. Further work on the determination of $D_{m}$ is now in progress.

\section{Acknowledgment}

The authors gratefully acknowledge Profes- 


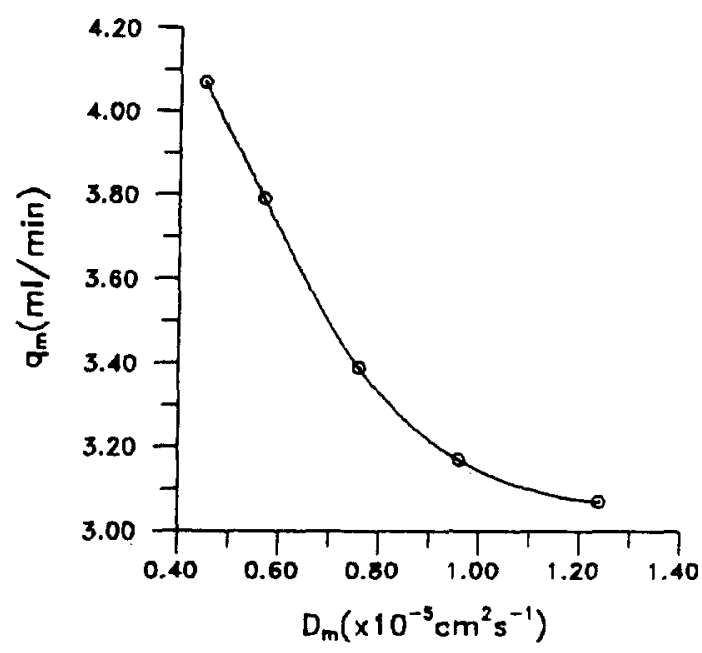

Fig. 7. The effect of $D_{\mathrm{m}}$ on $q_{\mathrm{m}}\left(V_{\text {inj }}=80 \mu \mathrm{l}\right.$, tube: $\left.L=233 \mathrm{~cm} ; d=0.5 \mathrm{~mm} ; R_{\mathrm{c}}=1.0 \mathrm{~cm}\right)$. sor E. H. Hansen for careful revision of the manuscript.

\section{References}

[1] J. Ruzicka and E.H. Hansen, Flow Injection Analysis, 2nd edn. Wiley, New York, 1988, p. 30.

[2] Y. Li and Y. Narusawa, Anal. Chim. Acta, 289 (1994) 355.

[3] M.A. Gomez-Nieto, M.D. Luque De Castro. A. Martin and M. Valcarcel, Talanta, 32 (1985) 319.

[4] J. Ruzicka and E.H. Hansen, Anal. Chim. Acta, 99 (1978) 37.

[5] G. Gerhardt and R.N. Adams, Anal. Chem., 54 (1982) 2618.

[6] A.J. Bard, Encyclopedia of Electrochemistry of the Elements, Vol. 2, Marcel Dekker, New York, 1974, p. 412.

[7] T. Korenaga, Anal. Chim. Acta, 261 (1992) 539. 